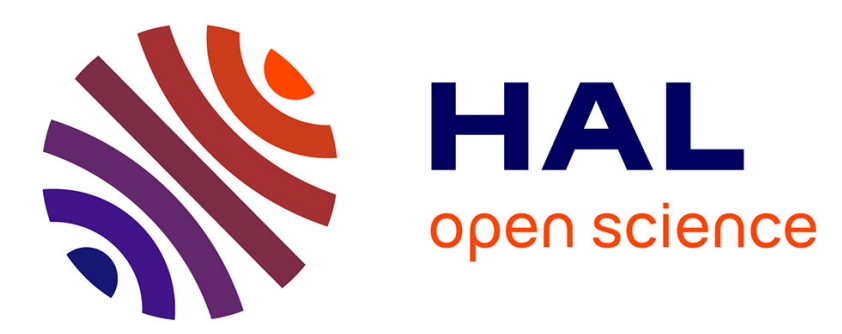

\title{
Normal form based analytical investigation of nonlinear power system dynamics under excitation
}

Tian Tian, Xavier Kestelyn, Olivier Thomas

\section{To cite this version:}

Tian Tian, Xavier Kestelyn, Olivier Thomas. Normal form based analytical investigation of nonlinear power system dynamics under excitation. 2017 IEEE Power \& Energy Society General Meeting (PESGM), Jul 2017, Chicago, United States. pp.1-5, 10.1109/PESGM.2017.8273920 . hal-02424297

\section{HAL Id: hal-02424297 \\ https://hal.science/hal-02424297}

Submitted on 27 Dec 2019

HAL is a multi-disciplinary open access archive for the deposit and dissemination of scientific research documents, whether they are published or not. The documents may come from teaching and research institutions in France or abroad, or from public or private research centers.
L'archive ouverte pluridisciplinaire HAL, est destinée au dépôt et à la diffusion de documents scientifiques de niveau recherche, publiés ou non, émanant des établissements d'enseignement et de recherche français ou étrangers, des laboratoires publics ou privés. 


\section{Normal Form based Analytical Investigation of Nonlinear Power System Dynamics under Excitation}

\author{
Tian TIAN, Xavier KESTELYN \\ Univ. Lille, Centrale Lille, Arts et Metiers Paris Tech, HEI, \\ EA 2697 - L2EP \\ Laboratoire d'Electrotechnique et d'Electronique de Puissance \\ F-59000 Lille, France
}

\author{
Olivier THOMAS \\ Arts et Metiers ParisTech \\ LSIS UMR CNRS 7296 \\ Laboratoire des Sciences de l'Information \\ et des Systemes \\ 8 bd. Louis XIV 59000 Lille, France
}

\begin{abstract}
The increase of power exchanges through long lines makes power systems exhibiting predominant nonlinear interarea or local oscillations, for which the conventional linear analysis fails to offer an accurate picture. Existing 2nd Order and 3rd Order Normal Form methods make possible to do analytical investigations of the nonlinear interactions by including higherorder terms. However, those methods can only study the power system dynamics under free oscillations, usually used to study the effect of disturbances apply to the state variables, e.g the post-fault cases. When the system is subject to excitation, as variable power references or variable loads, there is no available Normal Form method in the literature making possible to study the higher-order oscillations. In this paper, for the first time to the author's knowledge, a methodology based on the Normal Form theory is proposed to give an analytical description of power system nonlinear dynamic response of excited power systems. The proposed method makes possible to simplify the initial problem by decomposing the complex power system dynamics into a set of simpler normal dynamics. The proposed analytical normal dynamics are validated using time-domain simulations and open the way to new analyzing tools for power grids.
\end{abstract}

\section{INTRODUCTION}

\section{A. Oscillations in Interconnected Power Systems}

Nowadays power systems are composed of a collection of interconnected subsystems working in collaboration to supply a common load, such as groups of generators [1] or interconnected Voltage Sources Converters working in parallel [2]-[4]. One of the most important issue in large-scale interconnected power systems under stress is the oscillations in the power system dynamics [5]. As the oscillations are essentially caused by the modal interactions between the system components, they are called Modal Oscillations. The complexity of analyzing such oscillations comes from the strong coupling between the components of the system. Understanding of the essence of such oscillations is necessary to help stabilizing and controlling power systems.

In this sense, analytical analysis tools are developed for:

1) Dynamic performance analysis: suggesting an approximate analytical solution;

2) Modal structural analysis: how the system components interact with each other;

3) Stability analysis.

\section{B. Various Analysis Tools}

Among all the analytical methods, linear analysis or linearizing analysis is the most conventional analysis tool, such as small-signal analysis [1] and linear modal analysis [6]. These methods are based on the linearization of the inherently nonlinear power system around the operating point by including only the first-order terms of its Taylor's series expansion. The eigen-analysis is made to obtain analytical results for the system dynamic performance and to know about the system stability. Besides, modal analysis (based on the eigenvectors) provides an insight of the modal structure of a power system demonstrating how the components of the power system interact with each other. Based on the modal analysis, power system stabilizers can be located in the right place to stabilizer the whole system [1] ensuring then its small-signal stability.

The nonlinearity of oscillations increases in case of stressed tie line flow between large areas [7]. Later researches suggested that in certain cases, linear analysis techniques might not provide an accurate picture of the power system modal characteristics. From 1996 to 2005, numerous papers [8][15] have published advocating the Normal Form (NF) as an efficient way for studying higher order modal interactions by inclusion of 2 nd order terms [7]. The applications are well developed, such as for example the optimal placement of stabilizers [16].

The increasing number of renewable-energy-basedgenerators, leading to unbalanced energy generation and presence of weak grids, associated to fast control devices [2], increases the nonlinear behavior of the system leading to the necessity of including 3rd order terms in the modal analysis. Several explorations [3], [4], [17], [18] have been done, but their applications haven't been fully developed. Among them, methods [3], [4] are advancements of a method validated by physical experiments in beam structure analysis [19]-[21].

A method between the 2nd order and 3rd order NF analysis is proposed to suggest stability bounds [22] when the linear analysis fails to accurately identify them.

\section{Problematic}

Nonlinear analysis can replace the linear analysis as it does a better job in all the three issues: describing dynamic 
response, modal interaction and stability analysis. However, the existing nonlinear analysis tools only deal with the case of free oscillations (e.g the post-fault case after the fault is cleared), and not with excited systems ( i.e the oscillations caused by variable power references or variable loads). This largely limits the scope of the NF analysis. This is a big disadvantage compared to the linear analysis, which can deal with the case when there are changes in the input (voltage reference, power reference, load, etc) as well as when there are no excitation (free oscillations).

\section{Originality}

In this paper, a method is introduced to study the power system nonlinear forced oscillations. To the author's knowledge, it is for the first time that an analytical analysis tool based on NF theory is proposed to analyze the nonlinear power system dynamic under excitation. This enlarges the scope of NF analysis. Moreover, all the benefits provided by second order based NF and linear analysis can be inherited.

This paper is organized as follows. In Section II, the NF theory is introduced and the general procedures are outlined. Based on this, the previous analysis tools are summarized and compared. In section III, the proposed method, based on NF theory, is outlined and the methodology is introduced. The case study and the obtained results are shown in Section VI. Conclusions are given in Section V.

\section{Literature REVIEW OF APPliCATIONS OF NORMAL FORM THEORY}

The method of NF is well established to tackle with systems having nonlinear coupled dynamics.

The equations of the system are firstly approximated by a Taylor's expansion series. The procedure can be summarized as [23]:

1 simplifying the linear part of a system by the use of a linear transformation;

2 simplifying the higher-order terms by NF transformations up to different orders. In this paper, order 2 and order 3 NF transformations;

3 simplifying the transformed system by neglecting some higher-order terms depending on the expected accuracy, some nonlinear terms are kept or neglected, leading to decoupled or invariant subsystems having normal dynamics and obtaining the decoupled or invariant normal dynamics;

4a making a nonlinear analysis based on the normal dynamics: higher-order modal interactions, stability bound or limit cycles, etc;

$4 \mathrm{~b}$ obtaining the solutions of the normal dynamics and reconstructing the original system variables.

In this paper, the proposed approximation, called FDNF3, is derived and compared to existing approximations as DNF3 [3], [4], NF3 [20], more classical nonlinear analysis NF2 and and the representative linear analysis(LNM).

Table I gives an overview of the different approximations and shows that only FDNF3 approximation covers the whole
TABLE I

COMPARISON OF EXISTING AND PROPOSED NORMAL FORM APPROXIMATIONS

\begin{tabular}{c|c|c|c|c} 
Method & Transformation & Decoupled/Invariant & Normal Dynamics & Free Oscillations / Excited systems \\
\hline LNM [6] & linear & decoupled & linear & free and forced \\
NF2 [7] & order 2 & decoupled & linear & free \\
NF3 [19], [21] & order 3 & invariant & order 3 & free \\
DNF3 [3] [4] & order 3 & decoupled & order 3 & free \\
FDNF3 & order 3 & decoupled & order 3 & free and forced \\
\hline
\end{tabular}

application range of power system dynamic analysis as the linear analysis tool does.

\section{THEORETICAL FORMULATION}

\section{A. Modeling of Power System Oscillations}

The oscillatory modes of power system can be modeled by (1). If the variables of the system are gathers in a $N$ dimensional vector $\boldsymbol{q}$, the motion equation writes as:

$$
M \ddot{q}+C \dot{q}+K \boldsymbol{q}+\boldsymbol{f}_{n l}(\boldsymbol{q})=\boldsymbol{F}
$$

where $M, C$ are constant diagonal inertia and damping matrices, whose values depend on the physical and controller parameters of the system. $\boldsymbol{K}$ and $\boldsymbol{f}_{\boldsymbol{n} \boldsymbol{l}}$ indicate the coupling between the variables, where $\boldsymbol{K}$ is a constant matrix including the linear terms and $f_{n l}$ gathers the nonlinear terms coming from the 2 nd and 3rd order terms of the Taylor's series. $\boldsymbol{F}$ is the excitation vector.

\section{B. Linear Transformation: Modal Expansion}

A modal expansion $\boldsymbol{q}(t)=\boldsymbol{\Phi}^{T} \boldsymbol{x}(t)$ can transform (1) into modal coordinates with decoupled linear part [6].

$$
\begin{aligned}
& \ddot{X}_{p}+2 \xi_{p} \omega_{p} \dot{X}_{p}+\omega_{p}^{2} X_{p} \\
& +\sum_{i=1}^{N} \sum_{j \geq i}^{N} g_{i j}^{p} X_{i} X_{j}+\sum_{i=1}^{N} \sum_{j \geq i}^{N} \sum_{k \geq j}^{N} h_{i j k}^{p} X_{i} X_{j} X_{k}=F_{p}
\end{aligned}
$$

where $\xi_{p}$ is the $p$-th modal damping ratio, and $g_{i j}^{p}$ and $h_{i j k}^{p}$ are quadratic and cubic nonlinearities coming from $\boldsymbol{f}_{\boldsymbol{n} l}$ Taylor's series expansion. Neglecting the nonlinear terms in (2), a linear model called LNM [6] is obtained and can be used both for analysis and control of multi-input systems.

\section{Normal Form Transformation in Modal Coordinates}

A normal form transformation is proposed in [19], [21], which reads, $\forall p=1 \ldots N$ :

$$
X_{p}=R_{p}+\mathcal{R}^{(3)}\left(R_{i}, S_{i}\right), \quad Y_{p}=S_{p}+\mathcal{S}^{(3)}\left(R_{i}, S_{i}\right),
$$

where $Y_{p}=\dot{X}_{p}, S_{p}=\dot{R}_{p}$ and $\mathcal{R}^{(3)}, \mathcal{S}^{(3)}$ are third order polynomials in $R_{i}$ ans $S_{i}$, fully defined in [19], [21] and are functions of the $g_{i j}^{p}$ and $h_{i j k}^{p}$ coefficients present in (2).

\section{Normal Dynamics}

Up to order 3 and keeping all the terms after the transformation, it leads to a set of invariant oscillators (if no internal resonance occurs) defined as NF3 [19], [21]. NF3 separates the nonlinear terms into cross-coupling terms and self-coupling terms. Neglecting the cross-coupling terms, the equations defining the normal dynamics are decoupled and defined as DNF3 and FDNF3. 
1) DNF3: Free-damped Oscillations

$$
\begin{aligned}
& \ddot{R}_{p}+2 \xi_{p} \omega_{p} \dot{R}_{p}+\omega_{p}^{2} R_{p} \\
& \quad+\left(h_{p p p}^{p}+A_{p p p}^{p}\right) R_{p}^{3}+B_{p p p}^{p} R_{p} \dot{R}_{p}^{2}+C_{p p p}^{p} R_{p}^{2} \dot{R}_{p}=0
\end{aligned}
$$

2) FDNF3: Systems under excitation

$$
\begin{aligned}
\dot{R}_{p}= & S_{p}-2 b_{p p}^{p} S_{p} F_{p}-c_{p p}^{p} F_{p} R_{p} \\
\dot{S}_{p}= & -\omega_{p}^{2} R_{p}-2 \xi_{p} \omega_{p} \dot{R}_{p} \\
& -\left(h_{p p p}^{p}+A_{p p p}^{p}\right) R_{p}^{3}-B_{p p p}^{p} R_{p} \dot{R}_{p}^{2}-C_{p p p}^{p} R_{p}^{2} \dot{R}_{p} \\
& +F_{p}-2 \beta_{p p}^{p} S_{p} F_{p}-2 \gamma_{p p}^{p} F_{p} R_{p}
\end{aligned}
$$

It is shown that, when $F$ is zero, (5) becomes (4). In other words, (4) is a specific case of (5) or (5) is a generalization of (4). Equation (5) shows that transforming variables $X-Y$ coordinates into $R-S$ coordinates will add coupling terms in the excitation vector $F$ ( $2 \beta_{p p}^{p} S_{p} F_{p}-2 \gamma_{p p}^{p} F_{p} R_{p}$ terms). The $\mathrm{NF}$ approximation decouples the state-variables but adds some coupling terms in the excitation vector components.

\section{E. Reconstructing the Results for the Original System}

Multi-scale calculating methods can be used to compute an approximate analytical solution of (4) and (5). From $R_{p}, S_{p}$ variables, $X_{p}, Y_{p}$ can be reconstructed using transformations (3). The efficiency of FDNF3 is validated by a case-study based on interconnected VSCs and compared to the classical linear analysis tool LNM and nonlinear analysis tool NF2.

\section{CASE-Study: InTerConnected VSCs}

\section{A. Test System}

Modern girds are more and more composed of renewable energy based power generators that can be interconnected to the grid through long transmission lines [24]. This weak grid configuration can lead to nonlinear oscillations [3], [25]. To illustrate this particular case, a test case composed of two interconnected VSCs to a transmission grid as been chosen, as shown in Fig.1.

$V S C_{1}$ and $V S C_{2}$ are interconnected by a short connection line having a reactance $X_{12}$ and are both connected to the transmission grid by long transmission lines having reactances $X_{1}, X_{2}$.

To study the nonlinear interactions under disturbances or variable references or loads, a detailed mathematical model is firsly made, which consists of the physical structure as well as the current, voltage and power loops. To design the power loop, since the conventional PLL controller can cause synchronization problems when facing connections using long transmission lines [26], the Virtual Synchronous MachineCase (VSM) control strategy is adopted [27].

For the chosen test case, the equivalent switching frequency of VSCs is set as $1700 \mathrm{~Hz}$ and the control loops are tune Case II with time constants as $T_{\text {current }}=5 \mathrm{~ms}, T_{\text {voltage }}=60 \mathrm{~ms}$, $T_{\text {power }}=1.47 \mathrm{~s}$. Large Volume Capacitor is installed to avoid the voltage collapse.

The electromagnetic model of one VSC is a set of 13th order equations [27], and the order of the overall system

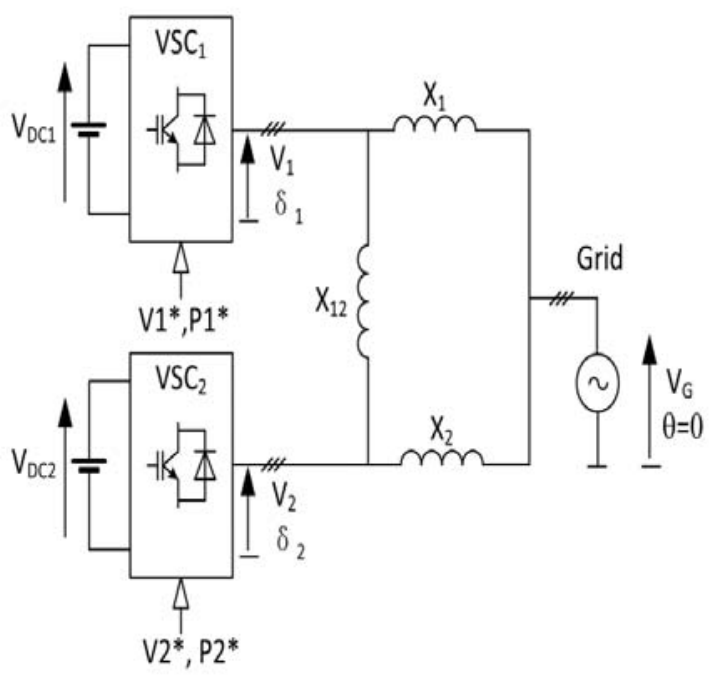

Fig. 1. Case test composed of interconnected VSCs

model can be higher than 26. Considering that the current and voltage loops are linear and that the time constants of the three loops respect $T_{\text {power }} \gg T_{\text {voltage }} \gg T_{\text {current }}$, the voltage $V_{1}$ and $V_{2}$ at the Point of Common Couplings (PCC) of VSCs are assumed sinusoidal with constant amplitudes and power flows are represented by dynamics in power angles. Thus, a reduced model to study the nonlinear interactions of the system presented in Fig.1, can be described as (6). A 4th order model is then chosen to govern the nonlinear dynamics of the system.

$$
\begin{aligned}
& M_{1} \frac{d^{2} \delta_{1}}{d t^{2}}+D_{1} \frac{d \delta_{1}}{d t}+\frac{V_{1} V_{g}}{X_{1}} \sin \delta_{1}+\frac{V_{1} V_{2}}{X_{12}} \sin \left(\delta_{1}-\delta_{2}\right)=P_{1}^{*} \\
& M_{2} \frac{d^{2} \delta_{2}}{d t^{2}}+D_{2} \frac{d \delta_{2}}{d t}+\frac{V_{2} V_{g}}{X_{2}} \sin \delta_{2}+\frac{V_{1} V_{2}}{X_{12}} \sin \left(\delta_{2}-\delta_{1}\right)=P_{2}^{*}
\end{aligned}
$$

In (6), $\delta_{1}$ and $\delta_{2}$ represent the power angles. $X_{1}$ and $X_{2}$ are the reactances of the transmission lines which connect the two VSCs to the transmission grid. $X_{12}$ is the reactance of the line interconnecting the two VSCs. $V_{1}, V_{2}, V_{g}$ are the RMS voltages at the PCC of the VSCs and at the grid. Finally, $P_{1}^{*}$ and $P_{2}^{*}$ are the active power references, which are set according to the expected operating points.

\section{B. Selected Case Study}

Two cases are selected.

: Post-fault case: the three phase fault happens near the terminal of $V S C_{2}$, the fault is cleared after $0.19 \mathrm{~s}$. The system experiences then free-damped oscillations.

I : Step Excitation in the Power Reference: $V S C_{1}$ and $V S C_{2}$ respectively transfer an amount of power as $0.68 \mathrm{pu}$ and $0.28 \mathrm{pu}$. It is supposed that there is an increase in the wind energy supply, which implies a step in the power reference of $0.2 \mathrm{pu}$ for both VSCs. The system experiences then oscillations due to excitation. 

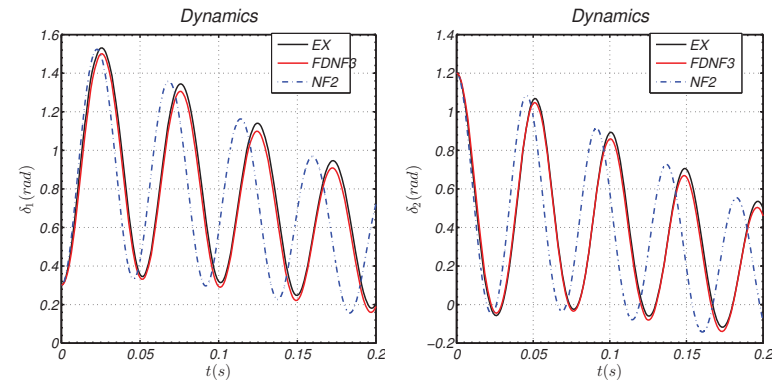

Fig. 2. Case I- System Dynamics under Free-damped Oscillations: $\delta_{1}$
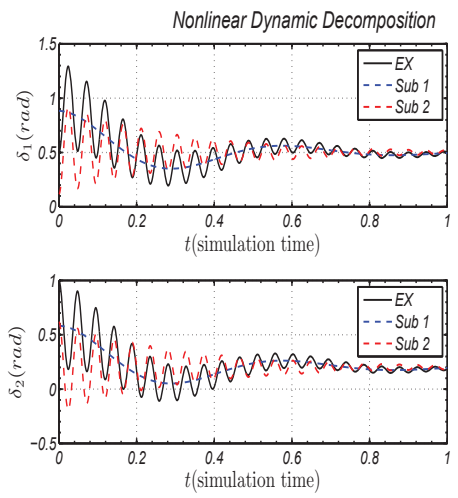

Fig. 3. Case I- System Dynamics under Free-damped Oscillations

As FDNF3 is a generalization of DNF3, for the sake of simplicity, FDNF3 is used for both the two cases and prove to be efficient in comparison with NF2 and LNM approaches.

\section{Analysis based on EMT Simulations}

The time-domain simulation is done by using a EMT software.

1) Original Results for Case I: The dynamics in the power angles of the VSCs, $\delta_{1}$ and $\delta_{2}$ are shown in Fig.2. Time $t=0$ is the time when the power angle reaches its maximum.

It is shown in Fig. 2 that FDNF3 can describe the nonlinear dynamics more accurately than NF2, not only in amplitude but also in frequency since it is based on a 3rd order Taylor's series terms, with a NF transformation up to 3rd order and higher-order terms kept in the normal dynamics.

FDNF3 has the property of decomposing a $N$-dimensional nonlinear system into a nonlinear sum of nonlinear 1dimensional nonlinear systems, analogous to linear analysis that decompose a $N$-dimensional linear system into a linear sum of 1-dimensional linear systems. In this way, the complex power dynamics can be reviewed as the combination simpler ones.

Fig. 3 shows that, as proposed by the classical linear analysis, the system dynamic can be decoupled into 2 simpler ones $E x \approx S u b 1+S u b 2$, making it possible to identify that $S u b 1$ has a lower frequency and $S u b 2$ has a much higher frequency.

2) Original Results for Case II: The dynamics in the power angles of the VSCs, $\delta_{1}$ and $\delta_{2}$ are shown in Fig.4. The step
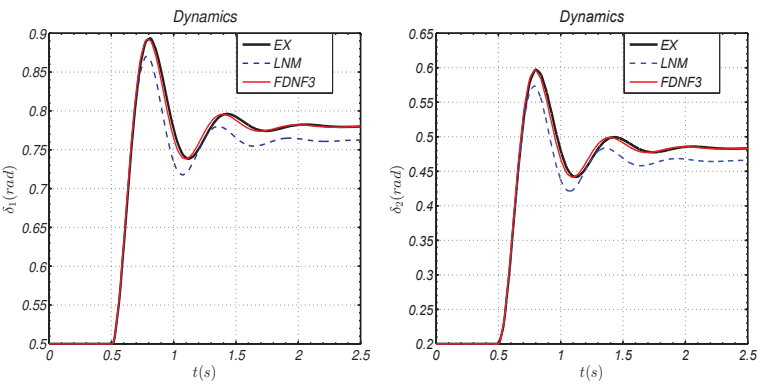

Fig. 4. Case II- System Dynamics under Step Excitation

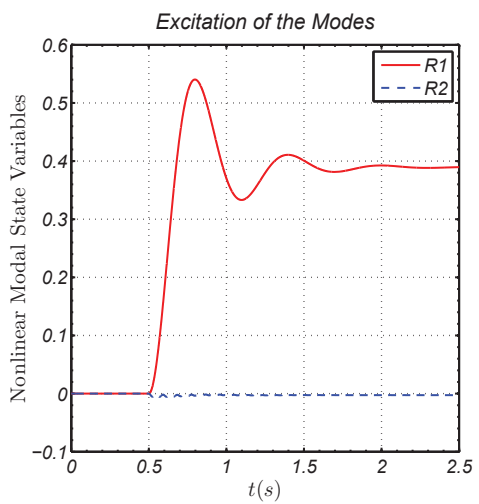

Fig. 5. Case II-Decomposition the Modes under Excitation: $R_{1}, R_{2}$

excitation in $P_{1}^{*}=P^{*}$ equal to $0.2 \mathrm{pu}$ happens at the time $t=0.5$.

It is shown in Fig.4 that FDNF3 can describe the system dynamic response much more accurately than the linear analysis LNM, especially the overshoot, which corresponds to the limit cycles [6] or the stability bound of system's nonlinear dynamics [22].

Also, as shown in Fig. 5, even both VSCs are excited, only one nonlinear mode is excited in $R-S$ coordinates. Therefore, inversely, by controlling $R 1, R 2$ we can make the decoupling control of $V S C_{1}$ and $V S C_{2}$ possible even if they work in the nonlinear region. It could be one way to overcome the difficulty on how to control VSCs independently when they work in nonlinear regions.

Transforming the original system dynamic into its normal dynamics gives a clear picture on how the excitation influences the system dynamics.

Compared to the classical linear analysis LNM and the nonlinear analysis NF2, the advantages of FDNF3 approach can be summarized as:

1) Describing more exactly the power system dynamics under disturbances or under excitation by decomposition of the complex system dynamics into simpler ones;

2) Making possible to independently control interconnected systems even if there are working in highly nonlinear regions. 
TABLE II

PARAMETERS OF THE CASE STUDY

\begin{tabular}{|l|c|c|}
\hline$M_{1}=3.2$ & $X_{1}(p u)=0.7$ & $V_{N}=400 k V$ \\
\hline$M_{2}=3.2$ & $X_{2}(p u)=0.7$ & $S_{N}=1 G M W$ \\
\hline$D_{1}=20$ & $X_{12}(p u)=0.01$ & $V_{1}=V_{2}=V_{g}=1 p u$ \\
\hline$D_{2}=20$ & $f_{g}=50 H z$ & \\
\hline
\end{tabular}

\section{CONCLUSION}

In this paper, to the author's knowledge, it is the first time that Normal Form theory is applied to study the power system dynamics under excitation (i.e. with variable references or loads). The proposed method is validated by comparing results with time-domain simulations based on an EMT software. For the sake of simplicity, only the results under the case $N=2$ are presented, but the possibility of working with large-scale problems will be investigated by the methodology suggested in this paper.

Although the chosen test case is composed of inteconnected VSCs, since the system model is the same as groups of generators working in parallel, the proposed methodology can be applied for studying the modal oscillations between classical generators working in parallel.

In this paper, the proposed method only shows its ability to describe the power system response by decomposing the complex dynamics. Further applications may be found in modal structural analysis under excitation or stability analysis. Future work can be composed of:

1) Controlling the two VSCs independently in the nonlinear $R-S$ coordinates, reducing the oscillations in the system dynamics;

2) Making the stability analysis under excitation to see the power transfer limit of power system under severe stress (working in the nonlinear domain).

\section{REFERENCES}

[1] P. Kundur, N. J. Balu, and M. G. Lauby, Power system stability and control. McGraw-hill New York, 1994, vol. 7.

[2] J. Quintero, V. Vittal, G. Heydt, and H. Zhang, "The impact of increased penetration of converter control-based generators on power system modes of oscillation," Power Systems, IEEE Transactions on, vol. 29 , no. 5, pp. 2248-2256, Sept 2014.

[3] T. Tian, X. Kestelyn, O. Thomas, and X. G. Guillaume Denis, "Analytical investigation of nonlinear interactions between voltage source converters interconnected to a transmission grid," in Power Energy Society General Meeting, 2016. PES '16. IEEE, July 2016, pp. 1-10.

[4] T. Tian, X. Kestelyn, and O. Thomas, "Third-order based analytical investigation of nonlinear interactions between voltage source converters interconnected to a transmission grid," in 2016 18th European Conference on Power Electronics and Applications (EPE'16 ECCE Europe), Sept 2016, pp. 1-10.

[5] V. Vittal, N. Bhatia, and A. A. Fouad, "Analysis of the inter-area mode phenomenon in power systems following large disturbances," IEEE Transactions on Power Systems, vol. 6, no. 4, pp. 1515-1521, Nov 1991.

[6] A. H. Nayfeh and D. T. Mook, Nonlinear oscillations. New-York: John Wiley \& sons, inc., 1979.

[7] J. Sanchez-Gasca, V. Vittal, M. Gibbard, A. Messina, D. Vowles, S. Liu, and U. Annakkage, "Inclusion of higher order terms for small-signal (modal) analysis: committee report-task force on assessing the need to include higher order terms for small-signal (modal) analysis," Power Systems, IEEE Transactions on, vol. 20, no. 4, pp. 1886-1904, Nov 2005.
[8] C.-M. Lin, V. Vittal, W. Kliemann, and A. Fouad, "Investigation of modal interaction and its effects on control performance in stressed power systems using normal forms of vector fields," Power Systems, IEEE Transactions on, vol. 11, no. 2, pp. 781-787, May 1996.

[9] Y. X. Ni, V. Vittal, W. Kliemann, and A. A. Fouad, "Nonlinear modal interaction in hvdc/ac power systems with dc power modulation," IEEE Transactions on Power Systems, vol. 11, no. 4, pp. 2011-2017, Nov 1996.

[10] J. Thapar, V. Vittal, W. Kliemann, and A. A. Fouad, "Application of the normal form of vector fields to predict interarea separation in power systems," IEEE Transactions on Power Systems, vol. 12, no. 2, pp. 844850, May 1997

[11] S. Saha, A. A. Fouad, W. H. Kliemann, and V. Vittal, "Stability boundary approximation of a power system using the real normal form of vector fields," IEEE Transactions on Power Systems, vol. 12, no. 2, pp. 797802, May 1997.

[12] S. K. Starrett and A. A. Fouad, "Nonlinear measures of mode-machine participation [transmission system stability]," IEEE Transactions on Power Systems, vol. 13, no. 2, pp. 389-394, May 1998.

[13] G. Jang, V. Vittal, and W. Kliemann, "Effect of nonlinear modal interaction on control performance: use of normal forms technique in control design. i. general theory and procedure," IEEE Transactions on Power Systems, vol. 13, no. 2, pp. 401-407, May 1998.

[14] V. Vittal, W. Kliemann, Y. X. Ni, D. G. Chapman, A. D. Silk, and D. J. Sobajic, "Determination of generator groupings for an islanding scheme in the manitoba hydro system using the method of normal forms," IEEE Transactions on Power Systems, vol. 13, no. 4, pp. 1345-1351, Nov 1998.

[15] S. Zhu, V. Vittal, and W. Kliemann, "Analyzing dynamic performance of power systems over parameter space using normal forms of vector fields-part i: identification of vulnerable regions," IEEE Transactions on Power Systems, vol. 16, no. 3, pp. 444-450, Aug 2001.

[16] S. Liu, A. R. Messina, and V. Vittal, "Assessing placement of controllers and nonlinear behavior using normal form analysis," IEEE Transactions on Power Systems, vol. 20, no. 3, pp. 1486-1495, Aug 2005.

[17] I. Martínez, A. Messina, and E. Barocio, "Perturbation analysis of power systems: effects of second- and third-order nonlinear terms on system dynamic behavior," Electric Power Systems Research, vol. 71, no. 2, pp. 159 - 167, 2004. [Online]. Available: http://www.sciencedirect.com/science/article/pii/S037877960400029X

[18] Q. Huang, Z. Wang, and C. Zhang, "Evaluation of the effect of modal interaction higher than 2nd order in small-signal analysis," in Power Energy Society General Meeting, 2009. PES '09. IEEE, July 2009.

[19] C. Touzé, O. Thomas, and A. Chaigne, "Hardening/softening behaviour in non-linear oscillations of structural systems using non-linear normal modes," Journal of Sound Vibration, vol. 273, no. 1-2, pp. 77-101, 2004.

[20] C. Touzé and M. Amabili, "Non-linear normal modes for damped geometrically non-linear systems: application to reduced-order modeling of harmonically forced structures," Journal of Sound and Vibration, vol. 298, no. 4-5, pp. 958-981, 2006.

[21] C. Touzé, "Normal form theory and nonlinear normal modes: theoretical settings and applications," in Modal Analysis of nonlinear Mechanical Systems, G. Kerschen, Ed. Springer Series CISM courses and lectures, vol. 555, ISBN 978-3-7091-1790-2, 2014.

[22] H. Amano, T. Kumano, and T. Inoue, "Nonlinear stability indexes of power swing oscillation using normal form analysis," IEEE Transactions on Power Systems, vol. 21, no. 2, pp. 825-834, May 2006.

[23] A. H. Nayfeh, A Method of Normal Forms. New-York: John Wiley \& sons, inc., 2011.

[24] C. L. Archer and M. Z.Jacobson, "Supplying baseload power and reducing transmission requirements by interconnecting wind farms," Journal of Applied Meteorology and Climatology, nov 2007.

[25] R. Piwko, N. Miller, J. Sanchez-Gasca, X. Yuan, R. Dai, and J. Lyons, "Integrating large wind farms into weak power grids with long transmission lines," in Power Electronics and Motion Control Conference, 2006. IPEMC 2006. CES/IEEE 5th International, Aug 2006.

[26] A. Egea-Alvarez, S. Fekriasl, F. Hassan, and O. Gomis-Bellmunt, "Advanced vector control for voltage source converters connected to weak grids," Power Systems, IEEE Transactions on, vol. 30, no. 6, pp. 3072-3081, Nov 2015.

[27] S. D'Arco, J. Suul, and O. Fosso, "Automatic tuning of cascaded controllers for power converters using eigenvalue parametric sensitivities," Industry Applications, IEEE Transactions on, vol. 51, no. 2, pp. 17431753, March 2015. 